\title{
Crosstalks Between Gut Microbiota and Vibrio Cholerae
}

\author{
Zixin Qin ${ }^{\dagger}$, Xiaoman Yang ${ }^{\dagger}$, Guozhong Chen ${ }^{\dagger}$, Chaiwoo Park and Zhi Liu* \\ Department of Biotechnology, College of Life Science and Technology, Huazhong University of Science and Technology, \\ Wuhan, China
}

Vibrio cholerae, the causative agent of cholera, could proliferate in aquatic environment and infect humans through contaminated food and water. Enormous microorganisms residing in human gastrointestinal tract establish a special microecological system, which immediately responds to the invasion of $V$. cholerae, through "colonization resistance" mechanisms, such as antimicrobial peptide production, nutrients competition, and intestinal barrier maintenances. Meanwhile, $V$. cholerae could quickly sense those signals

OPEN ACCESS

Edited by:

Yang Fu,

Southern University of Science and

Technology, China

Reviewed by:

Xiaohui Zhou,

University of Connecticut,

United States

Govind Vediyappan,

Kansas State University,

United States

*Correspondence:

Zhi Liu

zhiliu@hust.edu.cn

tThese authors have contributed equally to this work

Specialty section:

This article was submitted to Molecular Bacterial Pathogenesis,

a section of the journal

Frontiers in Cellular and Infection

Microbiology

Received: 12 July 2020

Accepted: 17 September 2020

Published: 23 October 2020

Citation:

Qin Z, Yang X, Chen G, Park C and Liu Z (2020) Crosstalks Between Gut

Microbiota and Vibrio Cholerae.

Front. Cell. Infect. Microbiol.

10:582554.

doi: $10.3389 /$ fcimb. 2020.582554 and modulate the expression of relevant genes to circumvent those stresses during infection, leading to successful colonization on the surface of small intestinal epithelial cells. In this review, we summarized the crosstalks profiles between gut microbiota and V. cholerae in the terms of Type VI Secretion System (T6SS), Quorum Sensing (QS), Reactive Oxygen Species (ROS)/pH stress, and Bioactive metabolites. These mechanisms can also be applied to molecular bacterial pathogenesis of other pathogens in host.

\section{Keywords: gut microbiota, Vibrio cholerae, T6SS, QS, ROS, pH, Bioactive metabolites}

\section{INTRODUCTION}

To date, the devastating diarrheal disease cholera pandemics have been occurred seven times, and it is still endemics in the world, responsible for up to 3 million cases and 100,000 deaths annually (Theriot and Petri, 2020). Vibrio cholerae is the causal organism of the disease cholera, usually infects humans through ingestion of contaminated water and food, colonizes on the surface of small intestine villi with the aid of toxin coregulated pilus (TCP), and then secretes cholera toxin (CT), causing watery diarrhea and vomiting that lead to severe dehydration and even death (Yoon and Waters, 2019). V. cholerae is gram-negative, curved and facultative bacterium, with a long unipolar flagellum. According to the serological characteristics of its surface O-antigens, $V$. cholerae has over 200 serotypes, in which only O1 serotype cause the cholera pandemics. Based on the genotypes, O1 $V$. cholerae are further classified into classical biotype and El Tor type (Kaper et al., 1995). Classical biotype was the causative agent of the first six cholera epidemics, while El Tor V. cholerae caused the seventh epidemic (Albert, 1994).

In 1894, Metchnikoff, the Russian Nobel laureate, claimed that cholera was a disease to humans due to the fact that the phenotype of human infections could not be precisely replicated in the infections of laboratory model animals (Ritchie and Waldor, 2009). He further speculated that experimental animals could not be infected with $V$. cholerae because of the presence of microorganisms in the gut and suggested that animal cubs could be used as an experimental model because of their significantly lower abundance of gut microbes. Indeed, the 3-5-day-old infant mouse is used as the most common model for $V$. cholerae pathogenesis research. However, there are enormous bacteria resided in host gastrointestinal environment, with the 10 times more 
population than those of host cell, those gut microbes provide "colonization resistance" against pathogen invasion. Gut microbiota can produce short-chain fatty acids, antibacterial substances, signal molecules, and bioactive metabolites to benefit host health and to defend pathogenic bacterial infection, such as $V$. cholerae (Ducarmon et al., 2019). Correspondingly, V. cholerae can also sense the change of environments and adjust gene expression to increase adaptability (Parker and Sperandio, 2009).

In recent years, an increasing number of researchers turned to study $V$. cholerae pathogenesis under the microbiota background. This review will elaborate the crosstalk profiles between gut microbiota and $V$. cholerae and reveal their synergistic and antagonistic effects from the following aspects: (1) Type VI Secretion System (T6SS), (2) Quorum Sensing (QS), (3) Reactive Oxygen Species (ROS) and $\mathrm{pH}$, (4) Bioactive metabolites. In this review, we aspire to shine the light on the gut microbiota modulation as a promising therapy for $V$. cholerae and related enteric pathogens infection.

\section{T6SS-DEPENDED CROSSTALK BETWEEN GUT MICROBIOTA AND V. CHOLERAE}

T6SS is a syringe-like protein apparatus, affects the physiological function of susceptible cells by injecting toxic effectors into the cells, including prokaryotes as well as eukaryotes, and even lysing susceptible cells (Pukatzki et al., 2006; Schwarz et al., 2010). Up to $25 \%$ of Gram-negative bacteria, including $V$. cholerae, have been reported to contain T6SS (Pukatzki et al., 2006; Bingle et al., 2008; Basler et al., 2012), The V. cholerae T6SS consists of the following components: substrate proteins (HisF, VasA, VasB, VasE, and VasJ) attached to the cell outer membrane via protein-to-protein linkages, secretion-promoting tubular sheath exoskeleton protein VipA/VipB (Basler et al., 2012; Broms et al., 2013), sheath protein-coated a tubule of hemolysin co-regulated proteins (Hcps), VgrG protein responsible for punching holes in receptor cells and effector toxin (Shneider et al., 2013; Cianfanelli et al., 2016). Until now, six T6SS effectors with corresponding functions were reported in $V$. cholerae, among them, TleV1 (lipase), TseH (amidase), the C-terminal domain of VgrG-3 (lysozyme) targeted to prokaryotic cells, the C-terminal domain of VgrG-1 (actin-crosslinking) attacked eukaryotes, and VasX (pore formation), TseL (lipase) acted on both prokaryotic and eukaryotic cells (Pukatzki et al., 2007; Miyata et al., 2011; Russell et al., 2011, 2013). Function of $V$. cholerae T6SS only performed when its VipA/VipB protein was contracted rather than extended (Figure 1). Susceptible strains can be attacked by T6SS with its toxic effectors, so they have evolved a number of protective mechanisms, such as the production of cognate immune proteins or extracellular polysaccharides (Dong et al., 2013; Fu et al., 2013; Toska et al., 2018). The protective mechanisms, while remaining to be further excavated, may explain why some gut microbes are less vulnerable to T6SS attacks (Fast et al., 2018). Overall, T6SS plays an important role in the interaction between gut microbiota and $V$. cholerae.

Gut microbiota can suppress the expression of $V$. cholerae T6SS by converting bile acids to de-conjugated bile acids through the production of bile salt hydrolase (BSH) (Bachmann et al., 2015; Alavi et al., 2020). While the antagonistic interaction of intestinal microbes with $V$. cholerae T6SS enhances the virulence of $V$. cholerae either in mouse (Zhao et al., 2018), rabbit (Fu et al., 2018) or Drosophila model (Fast et al., 2018, 2020), concomitantly, the regenerative function and cell differentiation of intestinal cell are inhibited, resulting in massive intestinal cells shedding and then the exacerbation of the cholera symptoms (Fast et al., 2018, 2020). However, the precise mechanism of interaction between gut microbiota and $V$. cholerae by T6SS is largely unknown and needs to be further explored.

Conversely, $V$. cholerae can use T6SS to enhance its own adaptation in the gut. $V$. cholerae T6SS has direct lethal effect on the Prokaryotic organisms such as Escherichia coli MG1655, Pseudomonas aeruginosa (Basler et al., 2012, 2013; Dong et al., 2013; Fu et al., 2013; Fast et al., 2018). It can also stimulate the peristalsis of intestinal epithelial cells for excluding gut microbiota, like Aeromonas veronii, to improve the efficiency of the colonization (Logan et al., 2018; Booth and Smith, 2020). This suggests that T6SS is a critical method of communication between gut microbiota and V. cholerae (Figure 1).

\section{QS-DEPENDED CROSSTALK BETWEEN GUT MICROBIOTA AND V. CHOLERAE}

QS is widely present in different bacteria and adjusts the behavioral changes of the entire population according to the cell density. Different gut microbiota produces different quorum sensing molecules. For example, Acyl-homoserine lactone (AHL) as autoinducer molecule is produced by gramnegative bacteria. Small-molecule polypeptides are produced by gram-positive bacteria. Both types of bacteria can produce the furanyl dibasic compound (2S, 4S)-2-methyl-2,3,3,4tetrahydroxytetrahydrofuran borate (AI-2). Intestinal microbiota also communicates with each other via quorum sensing signals, transforming intestinal pathogenic commensal bacteria into pathogenic bacteria, and thus impacts on host health (Kim et al., 2020). While $V$. cholerae itself can produce three QS signal molecules, including inter-species communication autoinducer AI-2 (Schauder et al., 2001; Chen et al., 2002), intra-genusspecific autoinducer (S)-3-hydroxytridecan-4-one (CAI-1) (Kelly et al., 2009) and 3,5-dimethylpyrazin-2-ol (DPO) (Herzog et al., 2019). V. cholerae adapts to the gut environment through QS signals either by influencing the expression of the primary regulator $h a p R$, or through the $v q m A$ pathway, which regulates a number of physiological pathways, such as the expression of virulence factors, biofilms, T6SS, the formation of natural transformation states, cell aggregation, and other behaviors (Miller et al., 2002; Zhu et al., 2002; Hammer and Bassler, 2003; Beyhan et al., 2007; Shikuma et al., 2009; Suckow et al., 2011; Lo Scrudato and Blokesch, 2012; Shao and Bassler, 2014; Hawver et al., 2016; Jemielita et al., 2018). It is not surprised that QS is important for communication between gut microbiota and $V$. cholerae. 


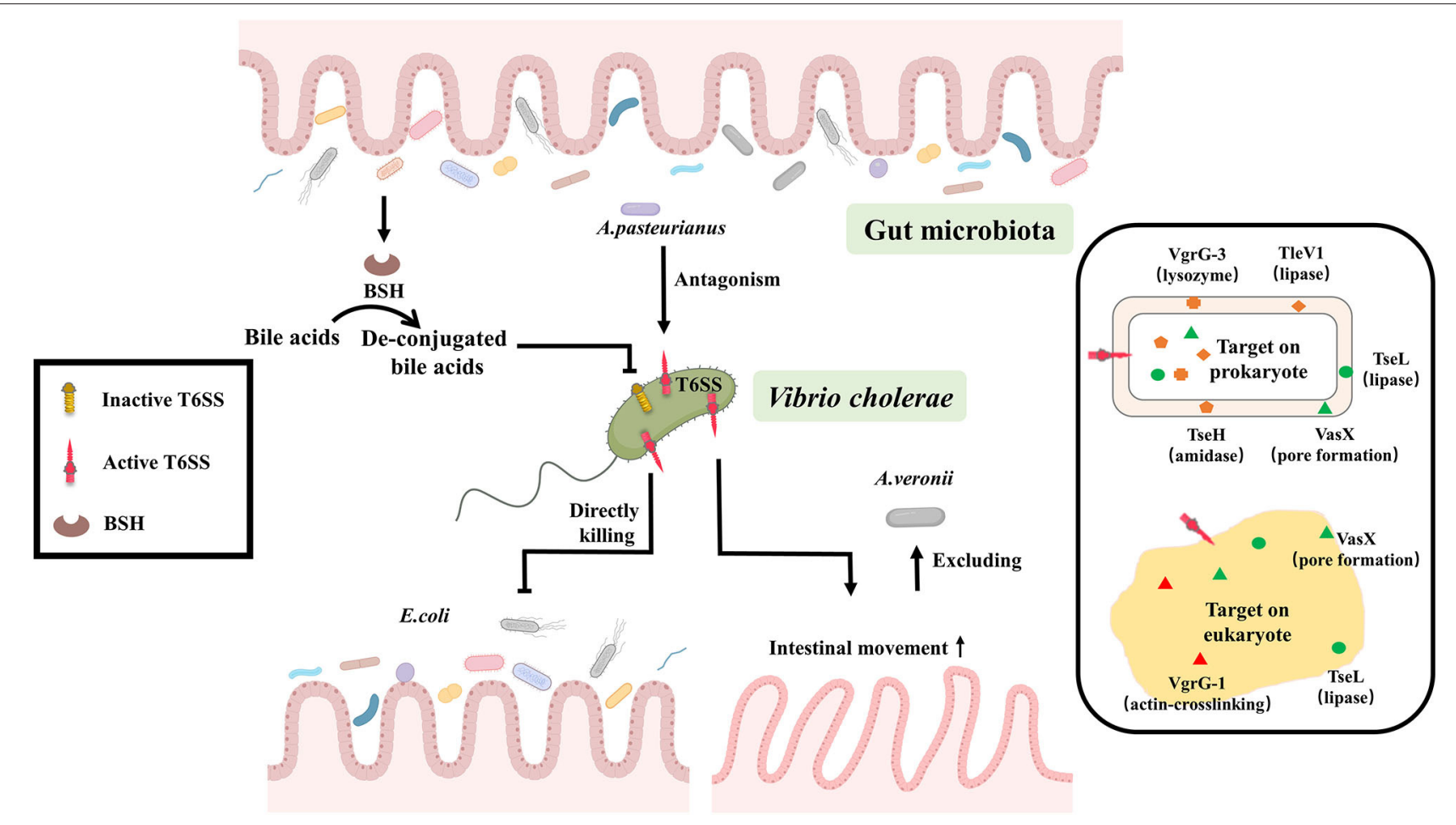

FIGURE 1 | T6SS-depended crosstalk between gut microbiota and $V$. cholerae. Gut microbiota can suppress the expression of $V$. cholerae T6SS by converting bile acids to de-conjugated bile acids through the production of $\mathrm{BSH}$, or some gut bacteria like $A$. pasteurianus can enhance the virulence of $V$. cholerae through antagonizing with $V$. cholerae T6SS. Conversely, V. cholerae T6SS has direct lethal effects on the prokaryote, such as Escherichia coli. Or it can stimulate the peristalsis of intestinal epithelial cells for excluding gut microbiota, like Aeromonas veronii, to improve the efficiency of the colonization. The effector of T6SS plays an important role in those reactions. Six T6SS effectors with corresponding functions were reported in V. cholerae, among them, TleV1 (lipase), TseH (amidase), the C-terminal domain of VgrG-3 (lysozyme) targeted to prokaryotic cells, the C-terminal domain of VgrG-1 (actin-crosslinking) attacked eukaryotes, and VasX (pore formation), TseL (lipase) acted on both prokaryotic and eukaryotic cells.

Gut microbiota can influence the physiological status of $V$. cholerae through QS. Ansel Hsiao found that the abundance of Ruminococcus obeum was significantly increased in the gut microbiota involved in recovery from $V$. cholerae infection. Further analysis of the function of $R$. obeum on $V$. cholerae revealed that the AI-2 signal produced by $R$. obeum significantly enhanced the colonization of $V$. cholerae. Interestingly, the AI-2 synthesized by R. obeum luxS does not act through the $V$. cholerae AI-2 sensor, LuxP, but rather affects the expression of $V$. cholerae virulence through another pathway that involves high expression of $v q m A$ (Hsiao et al., 2014). Some researchers speculated that this phenotype may be related to the accumulation of signal molecule DPO, which can activate the QS system via vqmA pathway, but the actual mechanism is controversial (Papenfort et al., 2017). Also, recently reported intestinal metabolite ethanolamine, is recognized by CqsR and then up-regulate the expression of hapR (Watve et al., 2020), can activate the QS system of $V$. cholerae. It shows that the QS signals produced by gut microbiota are complex and diverse, and it remains to be discovered if there is any communication of other group-sensing signals between gut microbiota and $V$. cholerae.

On the contrary, QS signals produced by $V$. cholerae may also have an effect on the physiological function of other microorganisms. For example, the CAI-1 produced by $V$. cholerae enhances the expression of T3SS virulence of enteropathogenic Escherichia coli (EPEC) E2348/69 and subsequent diarrhea in the host (Gorelik et al., 2019). Also, the $V$. cholerae QS system is still poorly understood, so there may be other factors by which $V$. cholerae communicates with gut microbiota via QS signals.

Because QS plays a critical role in interbacterial communication, the combination of QS signaling and synthetic biology methods to modify intestinal probiotics for detection, prevention, and treatment of $V$. cholerae infections has become a new therapeutic approach. For example, modified Escherichia coli Nissle1917 can produce $V$. cholerae-derived CAI-1 to reduce colonization of $V$. cholerae (Duan and March, 2008, 2010), or engineered Lactococcus lactis subsp. cremoris MG1363 can detect $V$. cholerae in the fecal through CAI-1 signals which is applied for the readily detection of $V$. cholerae (Higgins et al., 2007; Holowko et al., 2016; Mao et al., 2018). In summary, the $V$. cholerae QS will perform different functions depending on various environmental conditions. For $V$. cholerae, either external addition of CAI-1 chemicals (Higgins et al., 2007) or overexpression of CAI-1 using probiotics as carriers (Duan and March, 2008, 2010) inhibits the colonization of $V$. 
cholerae, whereas for other intestinal microorganisms, such as EPEC, CAI-1 activates their T3SS expression to enhance virulence. This also reflects the complexity and diversity of the gut environment. Therefore, better understanding of the $V$. cholerae QS system can provide a solid theoretical basis for the clinical treatment for cholera. It is becoming an increasingly promising therapy to mitigate and prevent the $V$. cholerae infection via interfering with the QS signal (Figure 2).

\section{ROS/PH-DEPENDED CROSSTALK BETWEEN GUT MICROBIOTA AND V. CHOLERAE}

Pathogens invading the intestinal environment primarily challenge intestinal innate immunity, which includes ROS and low $\mathrm{pH}$. It is reported that the diversity of the gut microbiota is related to the level of ROS (Yardeni et al., 2019). Gut microbiota can produce ROS by itself (Chen et al., 2019). Meanwhile, L-lactate produced by Lactobacillus plantarum can either mediate the consumption of NADH by NOX enzymes or be metabolized in mitochondria through the generation of pyruvate, enhancing ROS levels in the intestine (Iatsenko et al., 2018). Whereas, gut microbiota can also reduce ROS production by metabolizing Short-chain fatty acids (SCFAs) such as N-butyrate (Mottawea et al., 2016) or scavenge ROS by peroxidase (Yoon et al., 2016) to diminish ROS levels in the gut.

To better adapt to the intestinal environment, $V$. cholerae has also evolved a series of mechanisms to defend against or scavenge ROS, including the production of antioxidant molecule like glutathione (Meister and Anderson, 1983), catalase like $\mathrm{KatB} / \mathrm{KatG}$ (Xia et al., 2017), superoxide dismutase like OhrA/AphC (Cha et al., 2004; Liu et al., 2011, 2016; Wang et al., 2017), even it can protect itself from damaging by high levels of ROS in the intestinal environment through transforming its morphology, such as reversible phase variation between the rugose and smooth colony variants to response to ROS or biofilm formation (Faruque et al., 2006; Sengupta et al., 2016; Wang et al., 2018). Conversely, under inflammatory conditions $V$. cholerae impacts the structure and composition of intestinal microorganisms in multiple pathways. V. cholerae can produce a cholera toxin (CT), which causes electrolyte imbalance in the intestine, leading to diarrhea and thus disrupts the structure of intestinal commensal bacteria. Besides, under inflammatory conditions, the concentrations of $\mathrm{NO}_{3}^{-}$ increases and the available iron concentration decreases, while $V$. cholerae can make better use of $\mathrm{NO}_{3}^{-}$as a receptor for the electron respiratory chain and thus multiply faster and occupy an ecological niche (Bueno et al., 2018). V. cholerae also could promote their own proliferation by competing with gut microbiota and the host with iron (Rivera-Chavez and Mekalanos, 2019).

Apart from ROS, the host gut environment also has low $\mathrm{pH}$ pressure. $\mathrm{pH}$ is an important factor for bacterial growth in the gut. Gut microbiota can also alter $\mathrm{pH}$ to resist the invasion of $V$. cholerae. Culture supernatants of Lactobacillus lactis isolated from feces of healthy children inhibited the biofilm formation of $V$. cholerae. The phenotype that inhibited biofilm formation largely vanished after neutralization of the culture supernatant (Kaur et al., 2018). In addition, Escherichia coli 40 and Nissle 1917 isolated from the gut of healthy human volunteers were co-cultured with $V$. cholerae N16961 in LB medium containing glucose, respectively. It was found that both of them reduced the $\mathrm{pH}$ value in the medium and affected on the survival rate of $V$. cholerae (Sengupta et al., 2017). In the zebrafish model, the glucose combination with Escherichia coli 40 or Nissle 1917 reduced the colonization of $V$. cholerae $\mathrm{N} 16961$ by changing the $\mathrm{pH}$ in the gut (Nag et al., 2018). This is consistent with the use of glucose-based oral rehydration (ORS) combination with probiotic Escherichia coli during cholera treatment. These suggest that gut microbiota and their compositions can inhibit the colonization of $V$. cholerae by changing $\mathrm{pH}$.

Low $\mathrm{pH}$ in the intestinal microenvironment affects colonization of $V$. cholerae, and the reason may be that $V$. cholerae can respond to low $\mathrm{pH}$ by multiple mechanisms. For example, under hypoxic growth condition, $V$. cholerae can use nitrate as an oxidative phosphorylation electron acceptor, and adjust the process of nitrate/nitrite according to the environmental $\mathrm{pH}$, affecting its own adaptability by inhibiting glycolysis and proton motive force (PMF) (Bueno et al., 2018). In addition, NhaP1, as an antiporter of $\mathrm{K}^{+}\left(\mathrm{Na}^{+}\right) / \mathrm{H}^{+}$, enables $V$. cholerae to grow under low $\mathrm{pH}$ condition and maintain internal $\mathrm{pH}$ homeostasis by removing $\mathrm{K}^{+} / \mathrm{Na}^{+}$from the cytoplasm and ingesting $\mathrm{H}^{+}$. The $\mathrm{H}^{+}$enters the respiratory chain and is consumed. This mechanism is more suitable for $V$. cholerae to adapt intestinal microenvironment (Quinn et al., 2012). V. cholerae can also regulate lysine decarboxylase by $\mathrm{AphB}$ to consume $\mathrm{H}^{+}$so that it can alleviate low $\mathrm{pH}$ states (Kovacikova et al., 2010). Under alkaline conditions, $V$. cholerae can suppress related acid tolerance genes via OmpR and increase fitness (Kunkle et al., 2020). Indeed, $V$. cholerae itself has different $\mathrm{pH}$ patterns of fermentation depended on glucose. El Tor N16961 can produce 2, 3butanediol as the neutral product of fermentation, avoiding the acidification of the medium. In contrast, classic biotype O395 is unable to synthesize 2, 3-butanediol, therefore its viability is diminished during mixed fermentation with glucose due to the acidification of the medium by synthetic organic acids (Lee et al., 2020).

The mechanism by which $V$. cholerae affects gut microbiota by changing $\mathrm{pH}$ is unknown. However, $V$. cholerae can produce cholera toxin to cause intestinal inflammation of the host, resulting in intestinal electrolyte imbalance. This may affect $\mathrm{pH}$ changes to compete for niches.

Thus, it is known that gut microbiota can influence the ROS or $\mathrm{pH}$ of the gut environment to interfere with the colonization and infection of $V$. cholerae. In turn, $V$. cholerae can develop mechanisms to defend against it. This indicates that the interaction between gut microbiota and $V$. cholerae is complex and versatile (Figure 3 ). 


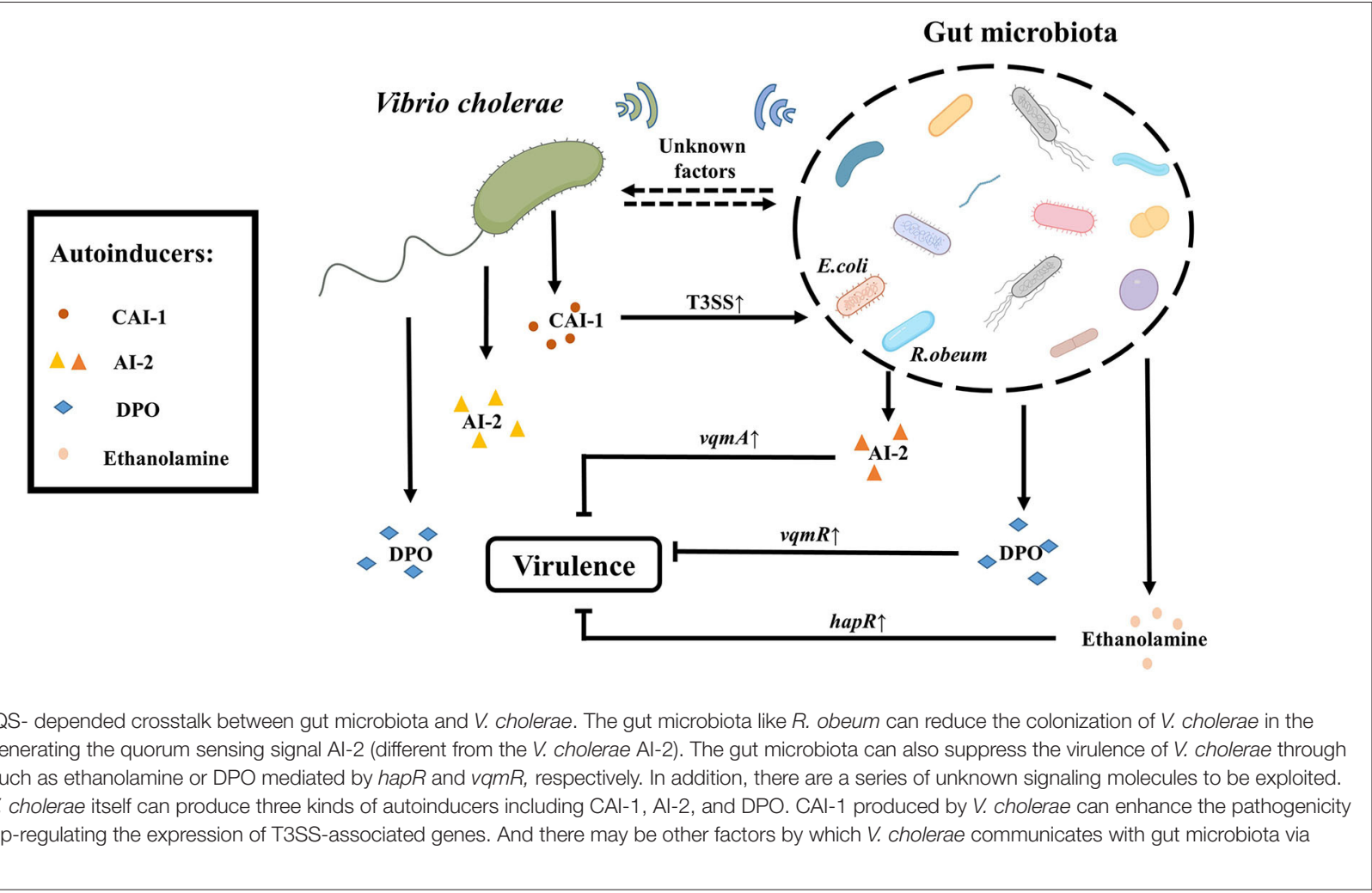

\section{BIOACTIVE METABOLITES-DEPENDED CROSSTALK BETWEEN GUT MICROBIOTA AND V. CHOLERAE}

Bioactive metabolites are also an important way for the gut microbiota to communicate with each other. The main bioactive metabolites include SCFAs, bacteriocins, and bile acids.

SCFAs are fatty acid fermentation products of microorganisms with indigestible polysaccharides as substrates. The concentration of SCFAs is relatively abundant in the proximal colon of the host. SCFAs are metabolites of gut microbiota and participate in the regulation of various host physiological process (Dalile et al., 2019). Surveillance of clinical samples of $V$. cholerae infection found that the content of SCFAs in the host decreased and the probiotic Bifidobacterium abundance decreased after $V$. cholerae infection. As the treatment progresses, the abundance of Bifidobacterium and SCFAs were return to normal levels (Monira et al., 2010). Besides, mice treated with clindamycin reduced the abundance of Bacteroides and the content of SCFAs to enhance the colonization ability of $V$. cholerae (You et al., 2019). All these indicate that probiotics in the host can antagonize the colonization of $V$. cholerae by secreting SCFAs. In the process of infecting the host, $V$. cholerae can also activate the transcription of acetyl-CoA synthase-1 (ACS-1) through the two-component system CrbRS, thereby regulating the conversion of $V$. cholerae acetate to deplete the acetate in the intestinal environment for protecting itself. The absence of this acetate will cause the host's insulin signal transduction pathway to be blocked and the accumulation of lipids, which will affect the host's health (Hang et al., 2014).

Antimicrobial peptide is a kind of peptide with antibacterial activity, which can be divided into two categories in the intestinal environment: host-derived antimicrobial peptides and microbialderived antimicrobial peptides (also known as bacteriocins). The host-derived antimicrobial peptides are mainly produced by intestinal epithelial cells and Pan's cells, including defensins, cathelicidins, lysozymes, chemokines, etc. (Chung and Raffatellu, 2019). During $V$. cholerae infection, the expression of hostderived antimicrobial peptides is upregulated, including $\alpha$ defensin (HD-5 and-6), $\beta$-defensin (hBD-1-4), cathelicidin (LL37), etc. (Qadri et al., 2004; Shirin et al., 2011). Human $\alpha$-defensin generally damages bacteria by disrupting cell membranes, while HD-6 defends against pathogenic bacteria by trapping microbes in the intestinal lumen (Chairatana and Nolan, 2017). Human $\beta$ defensin can also mediate membrane lysis to exert antimicrobial activity, as well as capture or kill bacteria by inducing self-nets and neutrophil extracellular traps (NETs) (Alvarez et al., 2018). In addition, cathelicidin can mediate membrane perturbation and induce ROS production to inhibit bacterial growth (RoweMagnus et al., 2019). V. cholerae has also evolved a response mechanism that uses the major virulence protein cholera toxin CT to activate several intracellular signaling pathways involving protein kinase A (PKA), ERK-MAPKinase, and Cox2 to downregulate the transcription of AMPs with CAMP accumulation (Chakraborty et al., 2008). The antimicrobial peptides or bacteriocin derived from bacteria can be divided 


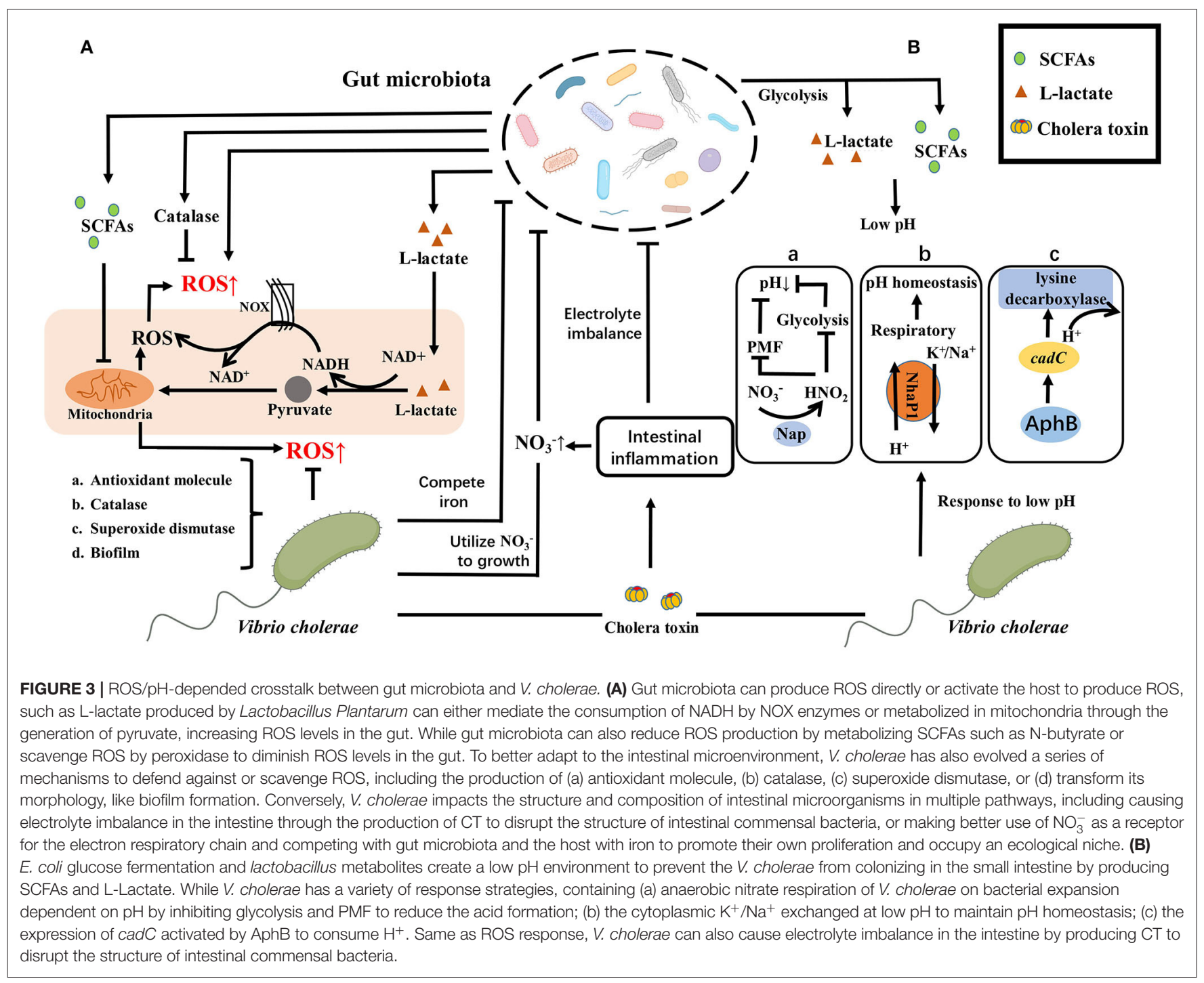

into gram-positive bacteriocin and gram-negative bacteriocin according to the type of bacteria. In the study of bacteriocin, Nisin produced by the genus streptococcus is relatively extensive (Hammami et al., 2013). The culture supernatant of Pediococcus acidilacticii QC38 isolated from food producing bacteriocin, showed inhibitory activity on the growth of $V$. cholerae in vitro, indicating that bacteriocins produced by probiotic QC38 had antagonistic effect on V. cholerae (Morales-Estrada et al., 2016). Previously, Lactobacillus casei OGM12 isolated from the food also showed inhibitory activity against $V$. cholerae, which produces bacteriocin casein A (Olasupo et al., 1995). Also, the bacteriocin produced by Streptococcus lactis 11451 showed inhibitory activity against $V$. cholerae in vitro (Spelhaug and Harlander, 1989). These indicate that the bacteriocin produced by probiotics has antibacterial activity against $V$. cholerae.

$V$. cholerae possesses its own antagonistic mechanism against this antimicrobial active peptide. Resistance-nodulation-division
(RND) mutants are sensitive to antimicrobial peptides in vitro and showed colonization defects on infant mice experiments. These phenotypes indicate RND efflux pump is important for $V$. cholerae to resist the toxicity of antimicrobial peptides (Bina et al., 2008). Besides, the outer membrane vesicles (OMV) of $V$. cholerae also play a crucial role in this stress condition. In the presence of antimicrobial peptides, the OMV content secreted by $V$. cholerae does not change, while the structure is altered, including the two outer membrane proteins OmpV and OmpW, as well as the Bap1 protein. Research showed that Bap1 protein can be combined with OmpT protein on the surface of OMV and can also be used as a ligand to capture antibacterial peptides to achieve the protection of bacteria (Duperthuy et al., 2013). V. cholerae also has a periplasmic space protein SipA, which can interact with the outer membrane protein OmpA. After the antibacterial peptide enters the periplasmic space, it may be captured by SipA, and 


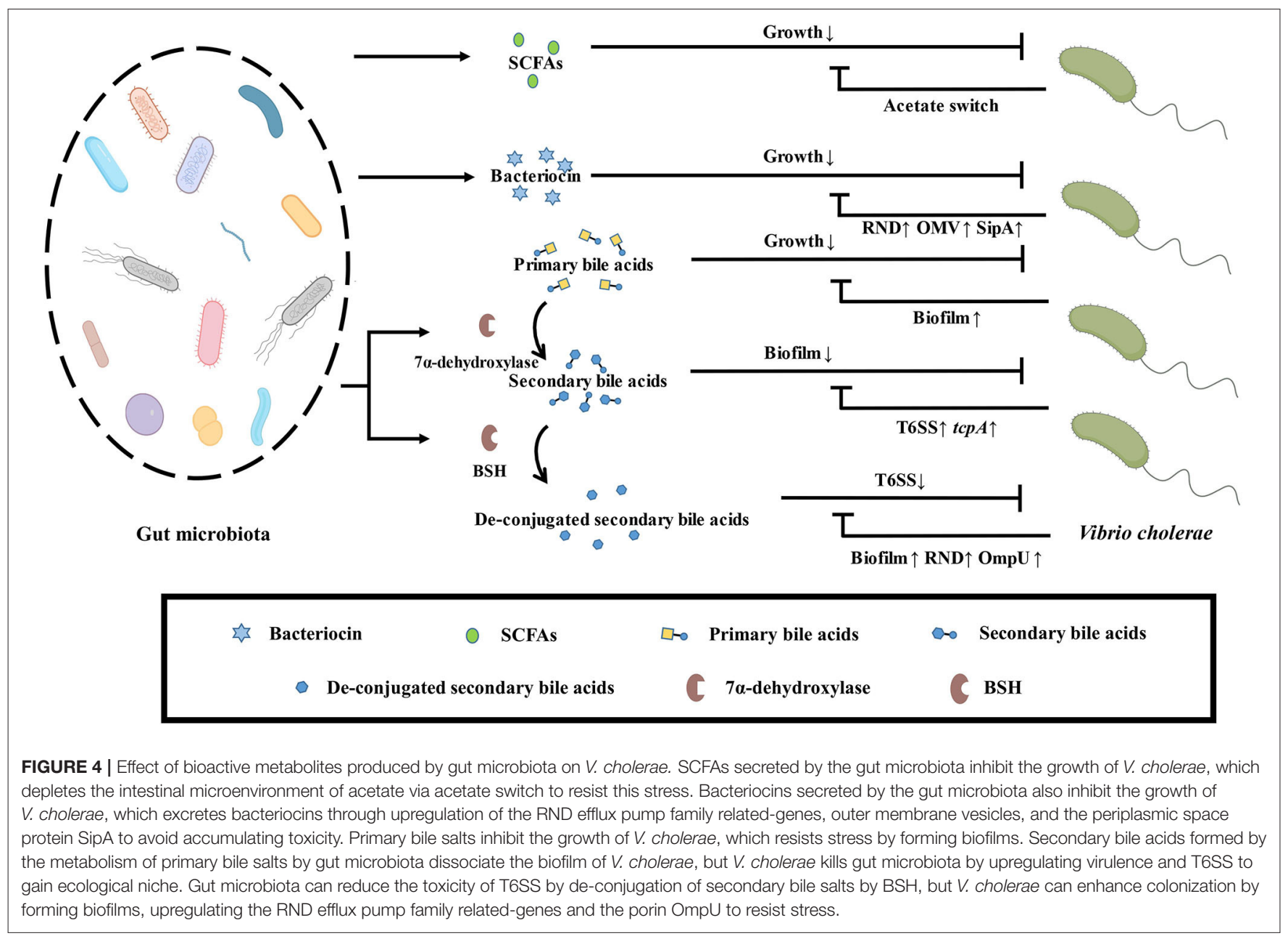

then interact with OmpA to transport out antimicrobial peptides (Saul-McBeth and Matson, 2019).

Bile acids are an important substance involved in the regulation of the hepatic-intestinal axis. Primary bile acids are produced from the body's liver using cholesterol as a substrate, followed by bile and discharged into the intestinal cavity, and then metabolized by the gut microbiota secondarily, in the terminal jejunum or ileum through the portal vein reabsorption back to the liver. In the human body, the primary bile acids produced by the liver mainly include cholic acid (CA) and chenodeoxycholic acid (CDCA), and then form conjugated bile acid (CBA) with taurine or glycine to enter the gallbladder and transport to small intestine (Ridlon et al., 2016). Most bile salts would be absorbed by the body from the terminal ileum, but some bile salts will be metabolized by gut microbiota to produce secondary bile salts (Wahlström et al., 2016). V. cholerae in the mature biofilm state is disrupted by taurocholate by altering the biofilm matrix and promoting biofilm disintegration (Hay and Zhu, 2015). Besides, taurocholate stimulates the formation of C207-C207 disulfide bonds between TcpP molecules, thereby activating the expression of the virulence gene toxT (Yang et al., 2013). In the case of mucin-activated $V$. cholerae T6SS expression, the taurine and glycine groups in the conjugated deoxycholic acid will enhance the killing effect of $V$. cholerae T6SS on intestinal commensal bacteria. Thus, while conjugated secondary bile acids inhibit $V$. cholerae biofilm formation, $V$. cholerae can also kill gut microbiota to gain ecological niche by increasing T6SS and virulence. The free deoxycholic acid metabolized by the gut microbiota in turn inhibits the toxicity of $V$. cholerae T6SS. Therefore, gut microbiota can resist the killing effect of pathogenic bacteria by adjusting bile acid metabolism (Bachmann et al., 2015). Pathogen-susceptible mice can acquire colonization resistance to $V$. cholerae by fecal microbiota transplantation (FMT). Further research has found that Blautia obeum's BSH enzyme deconjugate tcpA expression dependent secondary conjugated bile salts to down-regulate the expression of virulence gene in $V$. cholerae (Alavi et al., 2020). V. cholerae has reduced cell membrane permeability and upregulate $a c r A B$ gene expression to encode RND family outflow pump for avoiding accumulation toxicity of intracellular bile acids after sensing bile acid stimulation in vitro. This phenomenon is also found in rabbit isolated intestine models (Chatterjee et al., 2004). In addition, $V$. cholerae upregulates the porin OmpU and downregulates OmpT to resist deoxycholic acid stress via toxR (Provenzano 
and Klose, 2000; Ante et al., 2015). Biofilm-free V. cholerae activates $v p s$ gene and $v p s R$ transcriptional activator expression to form biofilms against bile acids toxicity after deoxycholic acid and cholic acid salt stimulation (Hung et al., 2006). Thus, while the gut microbiota can de-conjugate secondary conjugated bile salts to reduce $V$. cholerae T6SS and virulence, $V$. cholerae enhances colonization by forming biofilms, upregulating the RND efflux pump family proteins, and regulating porins against bile acids toxicity.

Probiotic flora abundance correlates with the concentration of SCFAs in the intestinal environment, and correspondingly, SCFAs resist $V$. cholerae infection. SCFAs, the metabolites involved in the regulation of many physiological functions, and their mechanism of protecting against $V$. cholerae infection are unclear. At present, the research on the antagonism of bacteriocin produced by gut microbiota to $V$. cholerae mainly focuses on the genus Streptococcus, and little research has been done on the production of bacteriocins by other commensal gut bacteria. Also, the research on the molecular mechanism of $V$. cholerae in response to antimicrobial peptides mainly uses host-derived antimicrobial peptides, and the interaction mechanism between $V$. cholerae and bacteriocins is also unclear. Studies on bile acids and $V$. cholerae have focused on the effects of bile acid salts conjugation and de-conjugation on the virulence of $V$. cholerae. In addition, bile acids are metabolized by BSH enzymes from the gut microbiota and then undergo metabolic pathways such as $7 \alpha-$ $\mathrm{HSDH}$ and $3 \alpha-\mathrm{HSDH}$ to produce other types of bile acids. The interaction of these kinds of bile acids with $V$. cholerae need more research (Figure 4).

\section{CONCLUSIONS AND PERSPECTIVES}

The virulence regulation network of $V$. cholerae has been wellstudied. However, the classical animal model and the germfree animal model of $V$. cholerae have ignored the role of gut microbiota. Interestingly, gut microbiota can not only use intestinal barrier to directly resist the invasion of $V$. cholerae, but also inhibit the colonization of $V$. cholerae through their metabolites, including autoinducer signaling molecules, antimicrobial peptides, short-chain fatty acids, bile salts and so on (Ducarmon et al., 2019). Correspondingly, V. cholerae, an intestinal pathogen, adjusts the expressions of its genes to respond to stress in the terms of T6SS, QS, ROS/pH, biofilm.

\section{REFERENCES}

Alavi, S., Mitchell, J. D., Cho, J. Y., Liu, R., Macbeth, J. C., and Hsiao, A. (2020). Interpersonal gut microbiome variation drives susceptibility and resistance to cholera infection. Cell 181, 1533-1546.e13. doi: 10.1016/j.cell.2020.05.036

Albert, M. J. (1994). Vibrio cholerae 0139 Bengal. J. Clin. Microbiol. 32, 2345-2349. doi: 10.1128/JCM.32.10.2345-2349. 1994

Alvarez, A. H., Martinez Velazquez, M., and Prado Montes de Oca, E. (2018). Human beta-defensin 1 update: potential clinical applications of the restless warrior. Int. J. Biochem. Cell. Biol. 104, 133-137. doi: 10.1016/j.biocel.2018.09.007
So far, the ways in which many QS signaling molecules produced by host or gut microbiota communicate with pathogens remain unknown, making it difficult to find novel quorum quenching molecules to reduce the pathogenicity of $V$. cholerae. Furthermore, traditional antibiotic therapy is facing a big challenge, which involves the drug resistance and the accelerating evolution of pathogens. Thus, the treatment of cholera by regulating the microecology of gut microbiota will be a potential therapy.

The intestine is a complex micro-ecological system, in which gut microbes have complex composition, with dynamic adjustment according to the external environment disturbance. So, the investigation of gut microbes and pathogen interactions require multidisciplinary technology platforms, including big data analysis, Next-generation sequencing, in situ fluorescence microscopic imagination, and lab-inchip automatic systems (Baumler and Sperandio, 2016). Gut microbiota is the important and active component in the gastrointestinal tract, which activity should be considered in studies of the pathogenesis of enteric pathogens, representatively, $V$. cholerae. The detailed information of molecular crosstalk between commensal gut bacteria and enteric pathogens would shed a light on the prevention and control of all infectious disease.

\section{AUTHOR CONTRIBUTIONS}

ZL provided the general concept. ZQ, XY, GC, and ZL drafted the initial concept of manuscript, and wrote the manuscript. CP provided the critical review of the manuscript. All the authors have seen and approved the final manuscript.

\section{FUNDING}

This work was supported by the National Key R\&D Program of China (2019YFA0905600), the National Natural Science Foundation of China (31770132 and 81873969).

\section{ACKNOWLEDGMENTS}

We thank all the members of Liu lab for helpful discussion, Quanxian She for critically reading the manuscript.

Ante, V. M., Bina, X. R., Howard, M. F., Sayeed, S., Taylor, D. L., and Bina, J. E. (2015). Vibrio cholerae leuO transcription is positively regulated by ToxR and contributes to bile resistance. J. Bacteriol. 197, 3499-3510. doi: 10.1128/JB.00419-15

Bachmann, V., Kostiuk, B., Unterweger, D., Diaz-Satizabal, L., Ogg, S., and Pukatzki, S. (2015). Bile salts modulate the mucinactivated type VI secretion system of pandemic Vibrio cholerae. PLoS Negl. Trop. Dis. 9:e0004031. doi: 10.1371/journal.pntd.00 04031

Basler, M., Ho, B. T., and Mekalanos, J. J. (2013). Tit-for-tat: type VI secretion system counterattack during bacterial cell-cell interactions. Cell 152, 884-894. doi: 10.1016/j.cell.2013.01.042 
Basler, M., Pilhofer, M., Henderson, G. P., Jensen, G. J., and Mekalanos, J. J. (2012). Type VI secretion requires a dynamic contractile phage tail-like structure. Nature 483, 182-186. doi: 10.1038/nature10846

Baumler, A. J., and Sperandio, V. (2016). Interactions between the microbiota and pathogenic bacteria in the gut. Nature 535, 85-93. doi: 10.1038/nature18849

Beyhan, S., Bilecen, K., Salama, S. R., Casper-Lindley, C., and Yildiz, F. H. (2007). Regulation of rugosity and biofilm formation in Vibrio cholerae: comparison of VpsT and VpsR regulons and epistasis analysis of $v p s T, v p s R$, and hapR. J. Bacteriol. 189, 388-402. doi: 10.1128/JB.00981-06

Bina, X. R., Provenzano, D., Nguyen, N., and Bina, J. E. (2008). Vibrio cholerae RND family efflux systems are required for antimicrobial resistance, optimal virulence factor production, and colonization of the infant mouse small intestine. Infect. Immun. 76, 3595-3605. doi: 10.1128/IAI.01620-07

Bingle, L. E., Bailey, C. M., and Pallen, M. J. (2008). Type VI secretion: a beginner's guide. Curr. Opin. Microbiol. 11, 3-8. doi: 10.1016/j.mib.2008.01.006

Booth, S. C., and Smith, W. P. J. (2020). Light sheets unveil host-microorganism interactions. Nat. Rev. Microbiol. 18:65. doi: 10.1038/s41579-019-0318-y

Broms, J. E., Ishikawa, T., Wai, S. N., and Sjostedt, A. (2013). A functional VipAVipB interaction is required for the type VI secretion system activity of Vibrio cholerae O1 strain A1552. BMC Microbiol. 13:96. doi: 10.1186/1471-2180-13-96

Bueno, E., Sit, B., Waldor, M. K., and Cava, F. (2018). Anaerobic nitrate reduction divergently governs population expansion of the enteropathogen Vibrio cholerae. Nat. Microbiol. 3, 1346-1353. doi: 10.1038/s41564-018-0253-0

Cha, M. K., Hong, S. K., Lee, D. S., and Kim, I. H. (2004). Vibrio cholerae thiol peroxidase-glutaredoxin fusion is a 2-Cys TSA/AhpC subfamily acting as a lipid hydroperoxide reductase. J. Biol. Chem. 279, 11035-11041. doi: $10.1074 /$ jbc.M312657200

Chairatana, P., and Nolan, E. M. (2017). Human alpha-defensin 6: a small peptide that self-assembles and protects the host by entangling microbes. Acc. Chem. Res. 50, 960-967. doi: 10.1021/acs.accounts.6b00653

Chakraborty, K., Ghosh, S., Koley, H., Mukhopadhyay, A. K., Ramamurthy, T., Saha, D. R., et al. (2008). Bacterial exotoxins downregulate cathelicidin (hCAP-18/LL-37) and human beta-defensin 1 (HBD-1) expression in the intestinal epithelial cells. Cell. Microbiol. 10, 2520-2537. doi: $10.1111 / j .1462-5822.2008 .01227 . x$

Chatterjee, A., Chaudhuri, S., Saha, G., Gupta, S., and Chowdhury, R. (2004). Effect of bile on the cell surface permeability barrier and efflux system of Vibrio cholerae. J. Bacteriol. 186, 6809-6814. doi: 10.1128/JB.186.20.6809-6814.2004

Chen, D., Wu, J., Jin, D., Wang, B., and Cao, H. (2019). Fecal microbiota transplantation in cancer management: current status and perspectives. Int. J. Cancer 145, 2021-2031. doi: 10.1002/ijc.32003

Chen, X., Schauder, S., Potier, N., Van Dorsselaer, A., Pelczer, I., Bassler, B. L., et al. (2002). Structural identification of a bacterial quorum-sensing signal containing boron. Nature 415, 545-549. doi: 10.1038/415545a

Chung, L. K., and Raffatellu, M. (2019). G.I. Pros: Antimicrobial defense in the gastrointestinal tract. Semin. Cell. Dev. Biol. 88, 129-137. doi: 10.1016/j.semcdb.2018.02.001

Cianfanelli, F. R., Alcoforado Diniz, J., Guo, M., De Cesare, V., Trost, M., and Coulthurst, S. J. (2016). VgrG and PAAR proteins define distinct versions of a functional type VI secretion system. PLoS Pathog. 12:e1005735. doi: 10.1371/journal.ppat.1005735

Dalile, B., Van Oudenhove, L., Vervliet, B., and Verbeke, K. (2019). The role of short-chain fatty acids in microbiota-gut-brain communication. Nat. Rev. Gastroenterol. Hepatol. 16, 461-478. doi: 10.1038/s41575-019-0157-3

Dong, T. G., Ho, B. T., Yoder-Himes, D. R., and Mekalanos, J. J. (2013). Identification of T6SS-dependent effector and immunity proteins by Tnseq in Vibrio cholerae. Proc. Natl. Acad. Sci. U.S.A. 110, 2623-2628. doi: 10.1073/pnas.1222783110

Duan, F., and March, J. C. (2008). Interrupting Vibrio cholerae infection of human epithelial cells with engineered commensal bacterial signaling. Biotechnol. Bioeng. 101, 128-134. doi: 10.1002/bit.21897

Duan, F., and March, J. C. (2010). Engineered bacterial communication prevents Vibrio cholerae virulence in an infant mouse model. Proc. Natl. Acad. Sci. U.S.A. 107, 11260-11264. doi: 10.1073/pnas.1001294107

Ducarmon, Q. R., Zwittink, R. D., Hornung, B. V. H., van Schaik, W., Young, V. B., and Kuijper, E. J. (2019). Gut microbiota and colonization resistance against bacterial enteric infection. Microbiol. Mol. Biol. Rev. 83:e00007-19. doi: 10.1128/MMBR.00007-19
Duperthuy, M., Sjostrom, A. E., Sabharwal, D., Damghani, F., Uhlin, B. E., and Wai, S. N. (2013). Role of the Vibrio cholerae matrix protein Bap1 in cross-resistance to antimicrobial peptides. PLoS Pathog. 9:e1003620. doi: 10.1371/journal.ppat.1003620

Faruque, S. M., Biswas, K., Udden, S. M., Ahmad, Q. S., Sack, D. A., Nair, G. B., et al. (2006). Transmissibility of cholera: in vivo-formed biofilms and their relationship to infectivity and persistence in the environment. Proc. Natl. Acad. Sci. U.S.A. 103, 6350-6355. doi: 10.1073/pnas.0601277103

Fast, D., Kostiuk, B., Foley, E., and Pukatzki, S. (2018). Commensal pathogen competition impacts host viability. Proc. Natl. Acad. Sci. U.S.A. 115, 7099-7104. doi: 10.1073/pnas.1802165115

Fast, D., Petkau, K., Ferguson, M., Shin, M., Galenza, A., Kostiuk, B., et al. (2020). Vibrio cholerae-symbiont interactions inhibit intestinal repair in drosophila. Cell Rep. 30, 1088-1100.e1085. doi: 10.1016/j.celrep.2019.12.094

Fu, Y., Ho, B. T., and Mekalanos, J. J. (2018). Tracking Vibrio cholerae cell-cell interactions during infection reveals bacterial population dynamics within intestinal microenvironments. Cell Host Microbe 23, 274-281.e272. doi: 10.1016/j.chom.2017.12.006

Fu, Y., Waldor, M. K., and Mekalanos, J. J. (2013). Tn-Seq analysis of Vibrio cholerae intestinal colonization reveals a role for T6SS-mediated antibacterial activity in the host. Cell Host Microbe 14, 652-663. doi: 10.1016/j.chom.2013.11.001

Gorelik, O., Levy, N., Shaulov, L., Yegodayev, K., Meijler, M. M., and Sal-Man, N. (2019). Vibrio cholerae autoinducer-1 enhances the virulence of enteropathogenic Escherichia coli. Sci. Rep. 9:4122. doi: 10.1038/s41598-019-40859-1

Hammami, R., Fernandez, B., Lacroix, C., and Fliss, I. (2013). Anti-infective properties of bacteriocins: an update. Cell. Mol. Life Sci. 70, 2947-2967. doi: 10.1007/s00018-012-1202-3

Hammer, B. K., and Bassler, B. L. (2003). Quorum sensing controls biofilm formation in Vibrio cholerae. Mol. Microbiol 50, 101-104. doi: 10.1046/j.1365-2958.2003.03688.x

Hang, S., Purdy, A. E., Robins, W. P., Wang, Z., Mandal, M., Chang, S., et al. (2014). The acetate switch of an intestinal pathogen disrupts host insulin signaling and lipid metabolism. Cell Host Microbe. 16, 592-604. doi: 10.1016/j.chom.2014.10.006

Hawver, L. A., Giulietti, J. M., Baleja, J. D., and Ng, W. L. (2016). Quorum sensing coordinates cooperative expression of pyruvate metabolism genes to maintain a sustainable environment for population stability. MBio 7:16. doi: $10.1128 / \mathrm{mBio} .01863-16$

Hay, A. J., and Zhu, J. (2015). Host intestinal signal-promoted biofilm dispersal induces Vibrio cholerae colonization. Infect. Immun. 83, 317-323. doi: 10.1128/IAI.02617-14

Herzog, R., Peschek, N., Fröhlich, K. S., Schumacher, K., and Papenfort, K. (2019). Three autoinducer molecules act in concert to control virulence gene expression in Vibrio cholerae. Nucleic Acids Res. 47, 3171-3183. doi: 10.1093/nar/gky1320

Higgins, D. A., Pomianek, M. E., Kraml, C. M., Taylor, R. K., Semmelhack, M. F., and Bassler, B. L. (2007). The major Vibrio cholerae autoinducer and its role in virulence factor production. Nature 450, 883-886. doi: 10.1038/nature06284

Holowko, M. B., Wang, H., Jayaraman, P., and Poh, C. L. (2016). Biosensing Vibrio cholerae with genetically engineered Escherichia coli. ACS Synth. Biol 5, 1275-1283. doi: 10.1021/acssynbio.6b00079

Hsiao, A., Ahmed, A. M., Subramanian, S., Griffin, N. W., Drewry, L. L., Petri, W. A., et al. (2014). Members of the human gut microbiota involved in recovery from Vibrio cholerae infection. Nature 515, 423-426. doi: 10.1038/nature13738

Hung, D. T., Zhu, J., Sturtevant, D., and Mekalanos, J. J. (2006). Bile acids stimulate biofilm formation in Vibrio cholerae. Mol. Microbiol. 59, 193-201. doi: 10.1111/j.1365-2958.2005.04846.x

Iatsenko, I., Boquete, J. P., and Lemaitre, B. (2018). Microbiota-derived lactate activates production of reactive oxygen species by the intestinal NADPH oxidase nox and shortens drosophila lifespan. Immunity 49, 929-942.e925. doi: 10.1016/j.immuni.2018.09.017

Jemielita, M., Wingreen, N. S., and Bassler, B. L. (2018). Quorum sensing controls Vibrio cholerae multicellular aggregate formation. Elife 7:e42057. doi: 10.7554/eLife.42057

Kaper, J. B., Morris, J. G. Jr., and Levine, M. M. (1995). Cholera. Clin. Microbiol. Rev. 8, 48-86. doi: 10.1128/CMR.8.1.48 
Kaur, S., Sharma, P., Kalia, N., Singh, J., and Kaur, S. (2018). Anti-biofilm properties of the fecal probiotic lactobacilli against vibrio spp. Front. Cell. Infect. Microbiol 8:120. doi: $10.3389 /$ fcimb. 2018.00120

Kelly, R. C., Bolitho, M. E., Higgins, D. A., Lu, W., Ng, W. L., Jeffrey, P. D., et al. (2009). The Vibrio cholerae quorum-sensing autoinducer CAI1: analysis of the biosynthetic enzyme CqsA. Nat. Chem. Biol 5, 891-895. doi: 10.1038/nchembio.237

Kim, E. K., Lee, K. A., Hyeon, D. Y., Kyung, M., Jun, K. Y., Seo, S. H., et al. (2020). Bacterial nucleoside catabolism controls quorum sensing and commensal-topathogen transition in the drosophila gut. Cell Host Microbe. 27, 345-357.e346. doi: 10.1016/j.chom.2020.01.025

Kovacikova, G., Lin, W., and Skorupski, K. (2010). The LysR-type virulence activator AphB regulates the expression of genes in Vibrio cholerae in response to low $\mathrm{pH}$ and anaerobiosis. J. Bacteriol. 192, 4181-4191. doi: 10.1128/JB.00193-10

Kunkle, D. E., Bina, X. R., and Bina, J. E. (2020). Vibrio cholerae OmpR contributes to virulence repression and fitness at alkaline pH. Infect. Immun 88:e00141-20. doi: 10.1128/IAI.00141-20

Lee, D., Kim, E. J., Baek, Y., Lee, J., Yoon, Y., Nair, G. B., et al. (2020). Alterations in glucose metabolism in Vibrio cholerae serogroup O1 El Tor biotype strains. Sci. Rep. 10:308. doi: 10.1038/s41598-019-57093-4

Liu, Z., Wang, H., Zhou, Z., Sheng, Y., Naseer, N., Kan, B., et al. (2016). Thiol-based switch mechanism of virulence regulator $\mathrm{AphB}$ modulates oxidative stress response in Vibrio cholerae. Mol. Microbiol. 102, 939-949. doi: $10.1111 / \mathrm{mmi} .13524$

Liu, Z., Yang, M., Peterfreund, G. L., Tsou, A. M., Selamoglu, N., Daldal, F., et al. (2011). Vibrio cholerae anaerobic induction of virulence gene expression is controlled by thiol-based switches of virulence regulator AphB. Proc. Natl. Acad. Sci. U.S.A. 108, 810-815. doi: 10.1073/pnas.1014640108

Lo Scrudato, M., and Blokesch, M. (2012). The regulatory network of natural competence and transformation of Vibrio cholerae. PLoS Genet. 8:e1002778. doi: 10.1371/journal.pgen.1002778

Logan, S. L., Thomas, J., Yan, J., Baker, R. P., Shields, D. S., Xavier, J. B., et al. (2018). The Vibrio cholerae type VI secretion system can modulate host intestinal mechanics to displace gut bacterial symbionts. Proc. Natl. Acad. Sci. U.S.A. 115, E3779-E3787. doi: 10.1073/pnas.1720133115

Mao, N., Cubillos-Ruiz, A., Cameron, D. E., and Collins, J. J. (2018). Probiotic strains detect and suppress cholera in mice. Sci. Transl. Med.10:eaao2586. doi: 10.1126/scitranslmed.aao2586

Meister, A., and Anderson, M. E. (1983). Glutathione. Annu. Rev. Biochem. 52, 711-760. doi: 10.1146/annurev.bi.52.070183.003431

Miller, M. B., Skorupski, K., Lenz, D. H., Taylor, R. K., and Bassler, B. L. (2002). Parallel quorum sensing systems converge to regulate virulence in Vibrio cholerae. Cell 110, 303-314. doi: 10.1016/S0092-8674(02)00829-2

Miyata, S. T., Kitaoka, M., Brooks, T. M., McAuley, S. B., and Pukatzki, S. (2011). Vibrio cholerae requires the type VI secretion system virulence factor VasX to kill Dictyostelium discoideum. Infect. Immun. 79, 2941-2949. doi: 10.1128/IAI.01266-10

Monira, S., Hoq, M. M., Chowdhury, A. K., Suau, A., Magne, F., Endtz, H. P., et al. (2010). Short-chain fatty acids and commensal microbiota in the faeces of severely malnourished children with cholera rehydrated with three different carbohydrates. Eur. J. Clin. Nutr. 64, 1116-1124. doi: 10.1038/ejcn.2010.123

Morales-Estrada, A. I., Lopez-Merino, A., Gutierrez-Mendez, N., Ruiz, E. A., and Contreras-Rodriguez, A. (2016). Partial characterization of bacteriocin produced by halotolerant pediococcus acidilactici strain QC38 isolated from traditional cotija Cheese. Pol. J. Microbiol. 65, 279-285. doi: $10.5604 / 17331331.1215607$

Mottawea, W., Chiang, C. K., Mühlbauer, M., Starr, A. E., Butcher, J., Abujamel, T., et al. (2016). Altered intestinal microbiota-host mitochondria crosstalk in new onset Crohn's disease. Nat. Commun. 7:13419. doi: 10.1038/ncomms13419

Nag, D., Breen, P., Raychaudhuri, S., and Withey, J. H. (2018). glucose metabolism by Escherichia coli inhibits vibrio cholerae intestinal colonization of zebrafish. Infect. Immun. 86:18. doi: 10.1128/IAI.00486-18

Olasupo, N. A., Olukoya, D. K., and Odunfa, S. A. (1995). Studies on bacteriocinogenic Lactobacillus isolates from selected Nigerian fermented foods. J. Basic Microbiol. 35, 319-324. doi: 10.1002/jobm.36203 50507
Papenfort, K., Silpe, J. E., Schramma, K. R., Cong, J. P., Seyedsayamdost, M. R., and Bassler, B. L. (2017). A Vibrio cholerae autoinducer-receptor pair that controls biofilm formation. Nat. Chem. Biol. 13, 551-557. doi: 10.1038/nchembio. 2336

Parker, C. T., and Sperandio, V. (2009). Cell-to-cell signalling during pathogenesis. Cell. Microbiol. 11, 363-369. doi: 10.1111/j.1462-5822.2008.01272.x

Provenzano, D., and Klose, K. E. (2000). Altered expression of the ToxR-regulated porins $\mathrm{OmpU}$ and $\mathrm{OmpT}$ diminishes Vibrio cholerae bile resistance, virulence factor expression, and intestinal colonization. Proc. Natl. Acad. Sci. U.S.A. 97, 10220-10224. doi: 10.1073/pnas.170219997

Pukatzki, S., Ma, A. T., Revel, A. T., Sturtevant, D., and Mekalanos, J. J. (2007). Type VI secretion system translocates a phage tail spike-like protein into target cells where it cross-links actin. Proc. Natl. Acad. Sci. U.S.A. 104, 15508-15513. doi: 10.1073/pnas.0706532104

Pukatzki, S., Ma, A. T., Sturtevant, D., Krastins, B., Sarracino, D., Nelson, W. C., et al. (2006). Identification of a conserved bacterial protein secretion system in Vibrio cholerae using the Dictyostelium host model system. Proc. Natl. Acad. Sci. U.S.A. 103, 1528-1533. doi: 10.1073/pnas.0510322103

Qadri, F., Bhuiyan, T. R., Dutta, K. K., Raqib, R., Alam, M. S., Alam, N. H., et al. (2004). Acute dehydrating disease caused by Vibrio cholerae serogroups O1 and $\mathrm{O} 139$ induce increases in innate cells and inflammatory mediators at the mucosal surface of the gut. Gut 53, 62-69. doi: 10.1136/gut.53.1.62

Quinn, M. J., Resch, C. T., Sun, J., Lind, E. J., Dibrov, P., and Hase, C. C. (2012). NhaP1 is a $\mathrm{K}+(\mathrm{Na}+) / \mathrm{H}+$ antiporter required for growth and internal $\mathrm{pH}$ homeostasis of Vibrio cholerae at low extracellular pH. Microbiology 158, 1094-1105. doi: 10.1099/mic.0.056119-0

Ridlon, J. M., Harris, S. C., Bhowmik, S., Kang, D. J., and Hylemon, P. B. (2016). Consequences of bile salt biotransformations by intestinal bacteria. Gut Microbes 7, 22-39. doi: 10.1080/19490976.2015.1127483

Ritchie, J. M., and Waldor, M. K. (2009). Vibrio cholerae interactions with the gastrointestinal tract: lessons from animal studies. Curr. Top. Microbiol. Immunol. 337, 37-59. doi: 10.1007/978-3-642-01846-6_2

Rivera-Chavez, F., and Mekalanos, J. J. (2019). Cholera toxin promotes pathogen acquisition of host-derived nutrients. Nature 572, 244-248. doi: 10.1038/s41586-019-1453-3

Rowe-Magnus, D. A., Kao, A. Y., Prieto, A. C., Pu, M., and Kao, C. (2019). Cathelicidin peptides restrict bacterial growth via membrane perturbation and induction of reactive oxygen species. MBio 10:19. doi: 10.1128/mBio.02021-19

Russell, A. B., Hood, R. D., Bui, N. K., LeRoux, M., Vollmer, W., and Mougous, J. D. (2011). Type VI secretion delivers bacteriolytic effectors to target cells. Nature 475, 343-347. doi: 10.1038/nature10244

Russell, A. B., LeRoux, M., Hathazi, K., Agnello, D. M., Ishikawa, T., Wiggins, P. A., et al. (2013). Diverse type VI secretion phospholipases are functionally plastic antibacterial effectors. Nature 496, 508-512. doi: 10.1038/nature12074

Saul-McBeth, J., and Matson, J. S. (2019). A periplasmic antimicrobial peptidebinding protein is required for stress survival in vibrio cholerae. Front. Microbiol. 10:161. doi: 10.3389/fmicb.2019.00161

Schauder, S., Shokat, K., Surette, M. G., and Bassler, B. L. (2001). The LuxS family of bacterial autoinducers: biosynthesis of a novel quorum-sensing signal molecule. Mol. Microbiol. 41, 463-476. doi: 10.1046/j.1365-2958.2001.02532.x

Schwarz, S., West, T. E., Boyer, F., Chiang, W. C., Carl, M. A., Hood, R. D., et al. (2010). Burkholderia type VI secretion systems have distinct roles in eukaryotic and bacterial cell interactions. PLoS Pathog. 6:e1001068. doi: 10.1371/journal.ppat.1001068

Sengupta, C., Ekka, M., Arora, S., Dhaware, P. D., Chowdhury, R., and Raychaudhuri, S. (2017). Cross feeding of glucose metabolism byproducts of Escherichia coli human gut isolates and probiotic strains affect survival of Vibrio cholerae. Gut Pathog. 9:3. doi: 10.1186/s13099-016-0153-x

Sengupta, C., Mukherjee, O., and Chowdhury, R. (2016). Adherence to Intestinal Cells Promotes Biofilm Formation in Vibrio cholerae. J. Infect. Dis. 214, 1571-1578. doi: 10.1093/infdis/jiw435

Shao, Y., and Bassler, B. L. (2014). Quorum regulatory small RNAs repress type VI secretion in Vibrio cholerae. Mol. Microbiol. 92, 921-930. doi: $10.1111 / \mathrm{mmi} .12599$

Shikuma, N. J., Fong, J. C., Odell, L. S., Perchuk, B. S., Laub, M. T., and Yildiz, F. H. (2009). Overexpression of VpsS, a hybrid sensor kinase, enhances biofilm formation in Vibrio cholerae. J. Bacteriol. 191, 5147-5158. doi: 10.1128/JB.004 01-09 
Shirin, T., Rahman, A., Danielsson, A., Uddin, T., Bhuyian, T. R., Sheikh, A., et al. (2011). Antimicrobial peptides in the duodenum at the acute and convalescent stages in patients with diarrhea due to Vibrio cholerae $\mathrm{O} 1$ or enterotoxigenic Escherichia coli infection. Microbes. Infect. 13, 1111-1120. doi: 10.1016/j.micinf.2011.0 6.014

Shneider, M. M., Buth, S. A., Ho, B. T., Basler, M., Mekalanos, J. J., and Leiman, P. G. (2013). PAAR-repeat proteins sharpen and diversify the type VI secretion system spike. Nature 500, 350-353. doi: 10.1038/nature12453

Spelhaug, S. R., and Harlander, S. K. (1989). Inhibition of foodborne bacterial pathogens by bacteriocins from Lactococcus lactis and Pediococcus pentosaceous (1). J. Food Prot. 52, 856-862. doi: 10.4315/0362-028X-52.12.856

Suckow, G., Seitz, P., and Blokesch, M. (2011). Quorum sensing contributes to natural transformation of Vibrio cholerae in a species-specific manner. $J$. Bacteriol. 193, 4914-4924. doi: 10.1128/JB.05396-11

Theriot, C. M., and Petri, W. A. Jr. (2020). Role of microbiota-derived bile acids in enteric infections. Cell 181, 1452-1454. doi: 10.1016/j.cell.2020. 05.033

Toska, J., Ho, B. T., and Mekalanos, J. J. (2018). Exopolysaccharide protects Vibrio cholerae from exogenous attacks by the type 6 secretion system. Proc. Natl. Acad. Sci. U.S.A. 115, 7997-8002. doi: 10.1073/pnas.180846 9115

Wahlström, A., Sayin, S. I., Marschall, H. U., and Bäckhed, F. (2016). Intestinal crosstalk between bile acids and microbiota and its impact on host metabolism. Cell Metab. 24, 41-50. doi: 10.1016/j.cmet.2016.05.005

Wang, H., Naseer, N., Chen, Y., Zhu, A. Y., Kuai, X., Galagedera, N., et al. (2017). OxyR2 Modulates OxyR1 activity and Vibrio cholerae oxidative stress response. Infect. Immun. 85:e0929-16. doi: 10.1128/IAI.009 29-16

Wang, H., Xing, X., Wang, J., Pang, B., Liu, M., Larios-Valencia, J., et al. (2018). Hypermutation-induced in vivo oxidative stress resistance enhances Vibrio cholerae host adaptation. PLoS Pathog 14:e1007413. doi: 10.1371/journal.ppat.1007413

Watve, S., Barrasso, K., Jung, S. A., Davis, K. J., Hawver, L. A., Khataokar, A., et al. (2020). Parallel quorum-sensing system in Vibrio cholerae prevents signal interference inside the host. PLoS Pathog 16:e1008313. doi: 10.1371/journal.ppat.100 8313
Xia, X., Larios-Valencia, J., Liu, Z., Xiang, F., Kan, B., Wang, H., et al. (2017). OxyR-activated expression of Dps is important for Vibrio cholerae oxidative stress resistance and pathogenesis. PLOS ONE 12:e0171201. doi: 10.1371/journal.pone.0171201

Yang, M., Liu, Z., Hughes, C., Stern, A. M., Wang, H., Zhong, Z., et al. (2013). Bile salt-induced intermolecular disulfide bond formation activates Vibrio cholerae virulence. Proc. Natl. Acad. Sci. U.S.A. 110, 2348-2353. doi: 10.1073/pnas.1218039110

Yardeni, T., Tanes, C. E., Bittinger, K., Mattei, L. M., Schaefer, P. M., Singh, L. N., et al. (2019). Host mitochondria influence gut microbiome diversity: A role for ROS. Sci. Signal 12:aaw3159. doi: 10.1126/scisignal.aaw3159

Yoon, M. Y., Min, K. B., Lee, K. M., Yoon, Y., Kim, Y., Oh, Y. T., et al. (2016). A single gene of a commensal microbe affects host susceptibility to enteric infection. Nat. Commun. 7:11606. doi: 10.1038/ncomms11606

Yoon, S. H., and Waters, C. M. (2019). Vibrio cholerae. Trends Microbiol. 27, 806-807. doi: 10.1016/j.tim.2019.03.005

You, J. S., Yong, J. H., Kim, G. H., Moon, S., Nam, K. T., Ryu, J. H., et al. (2019). Commensal-derived metabolites govern Vibrio cholerae pathogenesis in host intestine. Microbiome 7:132. doi: 10.1186/s40168-019-0746-y

Zhao, W., Caro, F., Robins, W., and Mekalanos, J. J. (2018). Antagonism toward the intestinal microbiota and its effect on Vibrio cholerae virulence. Science 359, 210-213. doi: 10.1126/science.aap 8775

Zhu, J., Miller, M. B., Vance, R. E., Dziejman, M., Bassler, B. L., and Mekalanos, J. J. (2002). Quorum-sensing regulators control virulence gene expression in Vibrio cholerae. Proc. Natl. Acad. Sci. U.S.A. 99, 3129-3134. doi: $10.1073 /$ pnas.052694299

Conflict of Interest: The authors declare that the research was conducted in the absence of any commercial or financial relationships that could be construed as a potential conflict of interest.

Copyright (c) 2020 Qin, Yang, Chen, Park and Liu. This is an open-access article distributed under the terms of the Creative Commons Attribution License (CC BY). The use, distribution or reproduction in other forums is permitted, provided the original author(s) and the copyright owner(s) are credited and that the original publication in this journal is cited, in accordance with accepted academic practice. No use, distribution or reproduction is permitted which does not comply with these terms. 